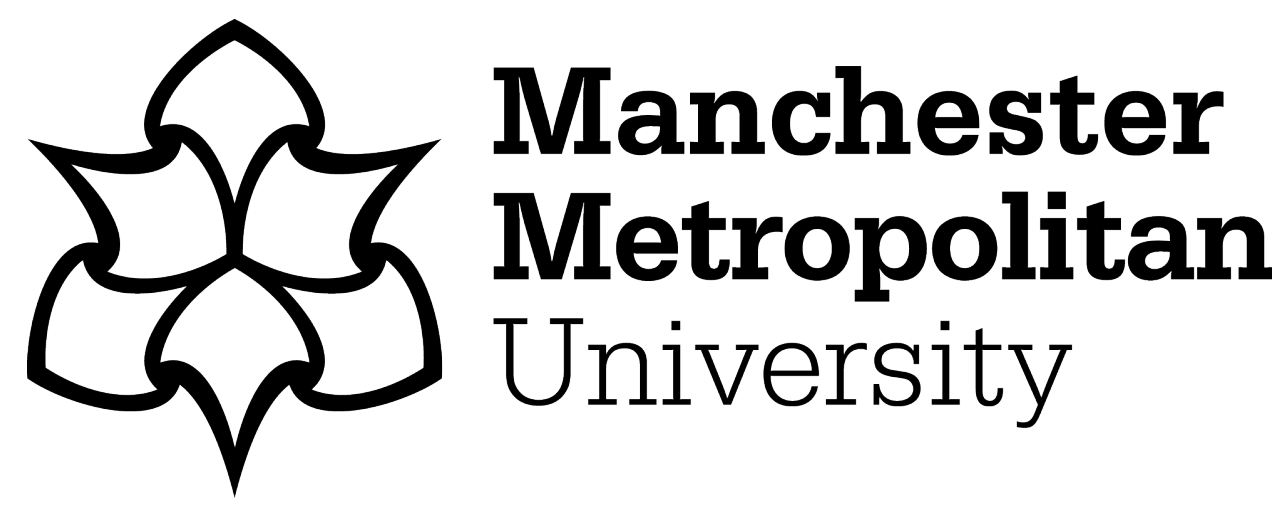

Ransley, E, Yan, S, Mai, T, Graham, D, Ma, Q, Musiedlak, PH, Engsig-Karup, AP, Eskilsson, C, Qian, Li ORCID logoORCID: https://orcid.org/0000-00029716-2342, Wang, J, Xie, Z, Sriram, V, Stoesser, T, Zhuang, Y, Li, Qi, Wan, D, Chen, G, Chen, Hao, Qian, Ling ORCID logoORCID: https://orcid.org/00000002-9716-2342, Ma, Zhihua ORCID logoORCID: https://orcid.org/00000002-2426-3038, Mingham, Clive, Causon, Derek ORCID logoORCID: https://orcid.org/0000-0003-1828-2168, Gatin, I, Jasak, H, Vukcevic, V, Downie, S, Higuera, P, Buldakov, E, Stagonas, D, Chen, Q, Zang, Jun and Greaves, D (2019) A Blind Comparative Study of Focused Wave Interactions with a Fixed FPSO-like Structure (CCP-WSI Blind Test Series 1). International Journal of Offshore and Polar Engineering (IJOPE), 29 (2). pp. 113127. ISSN 1053-5381

Downloaded from: https://e-space.mmu.ac.uk/622353/

Version: Accepted Version

Publisher: International Society of Offshore and Polar Engineers (ISOPE)

DOI: https://doi.org/10.17736/ijope.2019.jc748

Please cite the published version 


\title{
A blind comparative study of focused wave interactions with a fixed FPSO-like structure (CCP-WSI Blind Test Series 1)
}

\author{
E. Ransley ${ }^{1}$, S. Yan ${ }^{2}$, S. Brown ${ }^{1}$, T. Mai ${ }^{3}$, D. Graham ${ }^{1}$, Q. Ma ${ }^{2}$, P.-H. Musiedlak ${ }^{1}$, A. P. Engsig-Karup ${ }^{4}$, C. Eskilsson ${ }^{5}$, Qian Li ${ }^{2}$, \\ J. Wang ${ }^{2}$, Z. Xie, V. Sriram ${ }^{7}$, T. Stoesser ${ }^{14}$, Y. Zhuang ${ }^{8}$, Qi Li ${ }^{8}$, D. Wan ${ }^{8}$, G. Chen ${ }^{8}$, H. Chen ${ }^{9}$, L. Qian ${ }^{9}$, Z. Ma ${ }^{9}$, C. Mingham ${ }^{9}$,

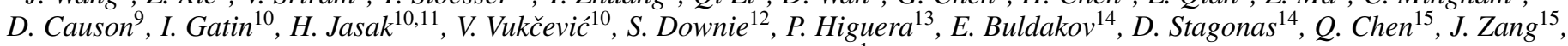 \\ D. Greaves ${ }^{1}$
}

1: University of Plymouth, UK; 2: City, University of London, UK; 3: National University of Civil Engineering, Vietnam; 4: Technical University of Denmark; 5: Aalborg University, Denmark; 6: Cardiff University, UK; 7: Indian Institute of Technology Madras; 8: Shanghai Jiao Tong University, China; 9: Manchester Metropolitan University, UK; 10: University of Zagreb, Croatia; 11: Wikki Ltd, UK; 12: Arup UK; 13: National University of Singapore; 14: University College London, UK; 15: University of Bath, UK

\begin{abstract}
Results from Blind Test Series 1, part of the Collaborative Computational Project in Wave Structure Interaction (CCP-WSI), are presented. Participants, with a range of numerical methods, simulate blindly the interaction between a fixed structure and focused waves ranging in steepness and direction. Numerical results are compared against corresponding physical data. The predictive capability of each method is assessed based on pressure and run-up measurements. In general, all methods perform well in the cases considered, however, there is notable variation in the results (even between similar methods). Recommendations are made for appropriate considerations and analysis in future comparative studies.
\end{abstract}

KEY WORDS: Code comparison; numerical validation; CFD; FNPT; PIC; hybrid codes; focused waves; range of steepness; range of incident wave angle; FPSO; run-up and pressure on bow.

\section{INTRODUCTION}

Numerical modelling has become an important process in the design of offshore structures and a vast number of tools have been developed as a consequence. The complete range of model fidelity is now available with the trade-off between computational efficiency and model complexity, i.e. the level of simplification made to the physics being solved, being a key driver when selecting a numerical tool for a particular application. Despite this, results from the 1st CCP-WSI Focus Group Workshop demonstrate that there still remain considerable uncertainties in the required level of model fidelity when using numerical methods to simulate the interaction of waves with offshore structures (CCP-WSI, 2016). Some progress, towards selecting a tool, can be made ahead of time by considering key parameters of the problem, such as the wave and geometric nonlinearity and corresponding dimensionless numbers. However, to promote the routine practical application of numerical tools, particularly high-fidelity methods, in industrial development processes, greater confidence in their applicability needs to be established.

The CCP-WSI Blind Test Workshops have been devised to improve the understanding of this issue and provide information for future development of numerical modelling standards. These workshops bring together numerical modellers from the wave structure interaction (WSI) community, and assess the numerical codes currently in use, by inviting participants to simulate a series of specific problems, covering a range of relevant complexities, without prior access to the physical measurements. The proposed test cases, in each Blind Test Series, are introduced at an introductory event, providing a forum for participants to discuss the cases and the validation process. All required information, to reproduce a set of bespoke physical validation experiments, is then made available in a release event; participants are then invited to submit simulation results for each case before a showcase event, in which the results from the test and present recommendations are shared with the community.

Comparative studies, over a broad range of test cases, are essential in finding a parametric understanding of the required model fidelity. The type of blind comparison discussed here is particularly valuable, in assessing the various strengths of numerical methods, as participants are not able to manipulate their results in light of the physical measurements. This encourages 'best practices' to be observed and offers a true representation of the capabilities of the method allowing for development of standard practices and certification of numerical models (crucial in encouraging significant uptake and derisking of their use by industry). Despite this, the process of performing a comparative study, with potentially vast amounts of multi-variant data submissions, is challenging (Hong et al., 2018) and established groups, from similar fields (NMRI, 2015), still rely on qualitative comparisons of specific cases with few offering a parametric understanding of predictive capability or consistent analysis methods between cases. Therefore, there is still value in producing evidence for best practices in comparative studies and recommendations relevant to future parametric certification protocols. 


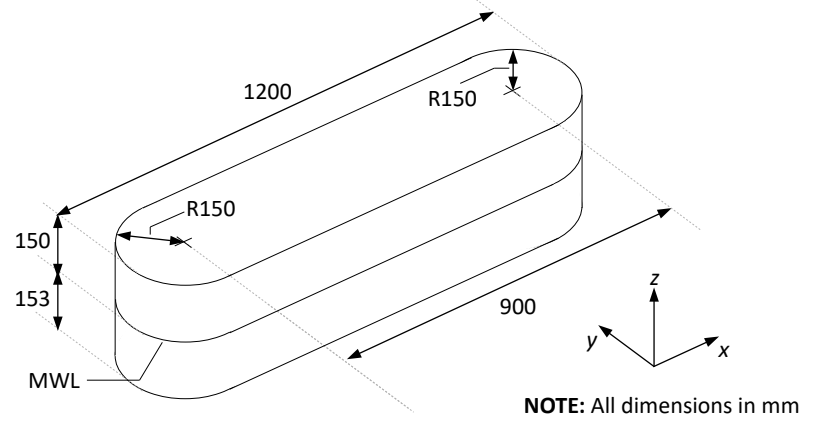

Fig. 1 Structure (M3) dimensions.

\section{CCP-WSI Blind Test Workshops - Series 1}

There will be a number of series' within the CCP-WSI Blind Test Workshops; Series 1 (the subject of this paper) is held in conjunction with the International Society of Offshore and Polar Engineers (ISOPE) conference, in collaboration with the International Hydrodynamics Committee (IHC); the introductory proposal was presented at the ISOPE conference in San Francisco, USA, on Wednesday 28th June 2017; the release of the Series 1 test cases was made on 12th October 2017, and; the showcase event was held over a series of special sessions at the 2018 ISOPE conference in Sapporo, Japan (10-15th June 2018). For more information on the CCP-WSI Blind Tests please visit the CCP-WSI website at http://www.ccp-wsi.ac.uk/?q=blind_test_workshops where supporting material is available including references to complimentary publications, photographs from the experiments and other related resources.

\section{TEST CASES}

The CCP-WSI Blind Test Series 1 test cases consist of a fixed, scalemodel, floating production storage and offloading (FPSO) vessel (known as M3 (Mai, et al. 2016)) subject to six focused wave events, with a range of steepness, $k A=0.13-0.21$ and incident angle, $\alpha=0^{\circ}, 10^{\circ} \&$ $20^{\circ}$, where $k$ is the wave number associated with the peak period, $T_{p}$, of the underlying energy spectrum of the wave and $A$ is the crest amplitude of the crest focused wave assuming linear superposition of the underlying wave components. All cases are free from violent flow features and correspond to non-breaking waves with no overturning of the free-surface or greenwater effects. The purpose of these particular test cases is to provide a parametric understanding, based on wave steepness and incident wave angle, of the predictive capabilities of a wide range of numerical WSI codes when assessing critical design factors, such as the pressure and run-up on FPSO hulls, and to evaluate the predictive capability as a function of the code complexity/execution time.

The structure (M3) has vertical sides and each end is semi-circular with the same radius $(0.15 \mathrm{~m})$. The full height of the structure is $0.303 \mathrm{~m}$, the length is $1.2 \mathrm{~m}$ and the draft is $0.153 \mathrm{~m}$ (Figure 1).

The Blind Test Series 1 test cases are split into two parts:

- Part 1 - considers waves of increasing steepness but constant angle of incidence, $\left(\alpha=0^{\circ}\right)$

- Part 2 - considers waves with increasing angle of incidence, $\alpha$, but constant steepness $(k A=0.17)$.

In each experiment, measurements of the pressure on the bow are recorded by an array of pressure transducers (sample frequency, $f_{s}$, $=1024 \mathrm{~Hz}$ ). The run-up, at various positions on the hull's surface, and the free-surface elevation in the vicinity of the structure are recorded by an array of resistive wave gauges $\left(f_{s}=128 \mathrm{~Hz}\right)$.

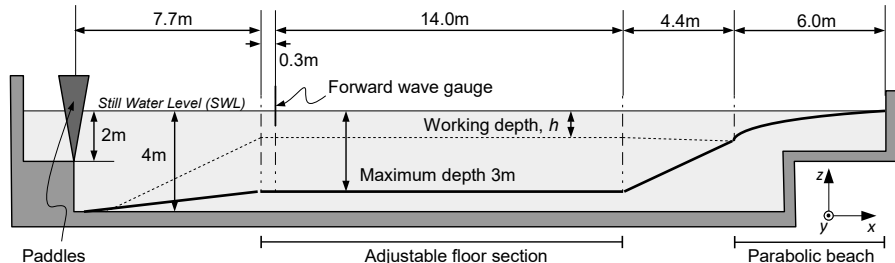

Fig. 2 Schematic of the COAST Laboratory Ocean Basin.

\section{Experimental Set-up}

Basin geometry

The experiments were performed in the Coastal, Ocean And Sediment Transport (COAST) Laboratory Ocean Basin ( $35 \mathrm{~m}$ long $\times 15.5 \mathrm{~m}$ wide) at the University of Plymouth, UK. The basin has 24 flap-type, forcefeedback-controlled wavemakers (hinge depth of $2 \mathrm{~m}$ ). The water depth at the wavemakers is $4 \mathrm{~m}$ and there is a linear slope to the working area where the water depth, $h$, was set to $2.93 \mathrm{~m}$. At the far end of the basin there is a parabolic absorbing beach (Figure 2).

Structure position and wave gauge layout - Part 1

In Part 1 the same wave gauge layout was utilised for both a series of empty tank tests and those with the structure in place (Figure 3).

Structure position and wave gauge layout - Part 2

In Part 2, two different arrays of wave gauges were used for the empty tank tests (Figure 4a) and those with the structure present (Figure 4b).

\section{Pressure sensor layout - Part 1}

For the cases in Part 1, an array of 6 pressure transducers were positioned on the bow of the FPSO on the centre-line (P1, P2 \& P3) and at $45^{\circ}$ to the port (P4, P5 \& P6) side; at the still water level and at depths of $\pm 0.05 \mathrm{~m}$ (Figure 5a).

\section{Pressure sensor layout - Part 2}

In Part 2, an array of 9 pressure transducers were positioned on the bow of the FPSO on the centre-line (P1, P2 \& P3) and at $45^{\circ}$ to the port (P7, P8 \& P9) and starboard (P4, P5 \& P6) sides; at the still water level and at depths of $\pm 0.05 \mathrm{~m}$ (Figure $5 \mathrm{~b}$ ).

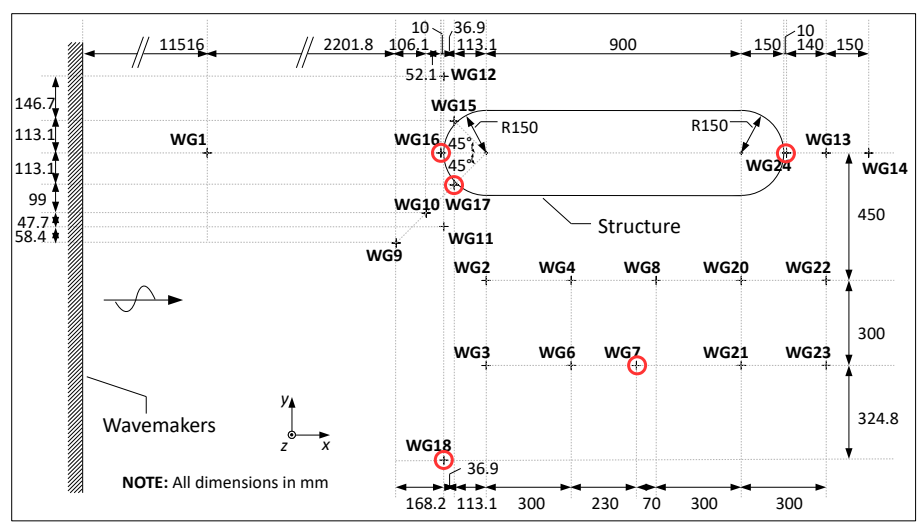

Fig. 3 Structure positioning and wave gauge layout for Part 1, for both the empty tank tests and with the structure in place. Positions highlighted with a red circle are those used for the comparative analysis. 

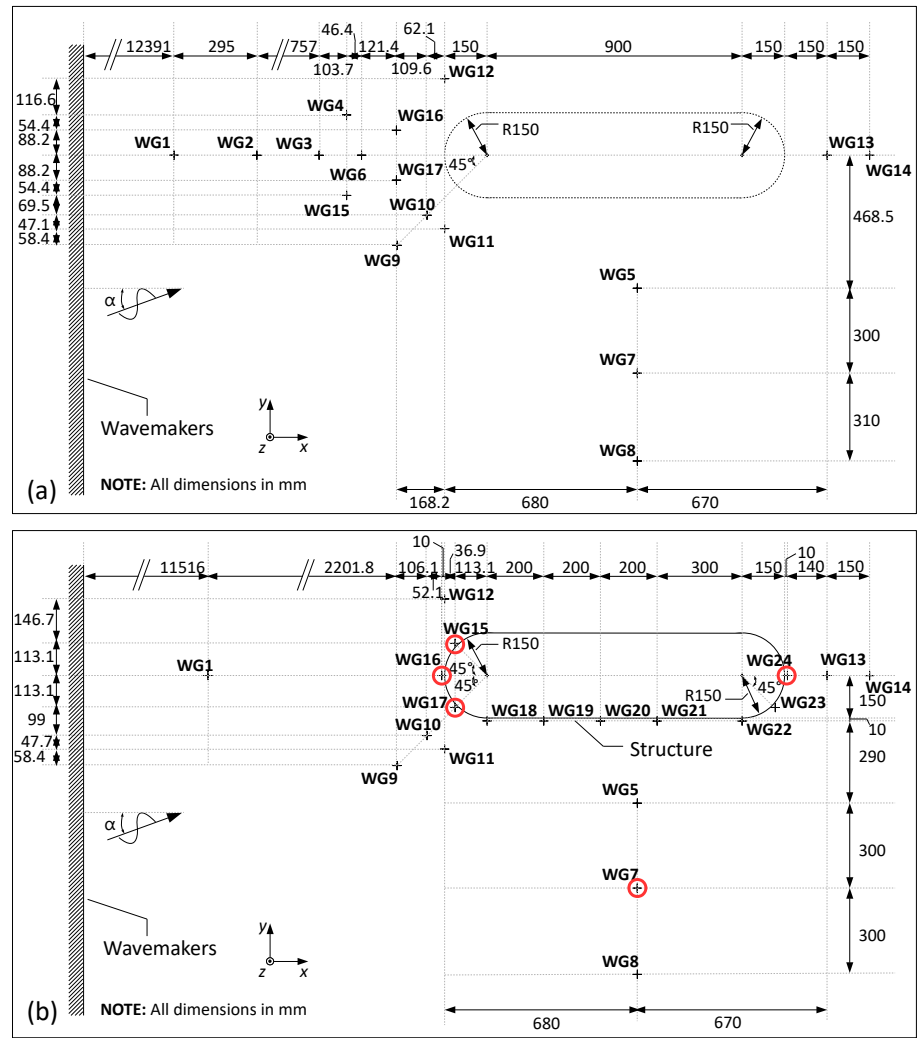

Fig. 4 Structure positioning and wave gauge layout for Part 2, for the empty tank tests (a) and with the structure (b). Positions highlighted with a red circle are those used for the comparative analysis.
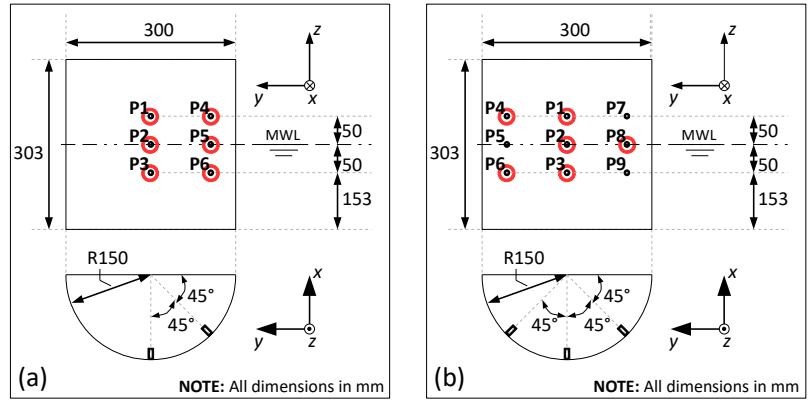

Fig. 5 Pressure probe layout on the bow of the FPSO for Part 1 (a), and; for Part 2 (b). Positions highlighted with a red circle are those used for the comparative analysis.

\section{Test Program}

For each test case, the incident waves were generated in the COAST Laboratory Ocean Basin (Figure 1) using the EDL paddle control software. The software is designed to reproduce the desired free-surface elevation by applying various corrections to account for the change in water depth in front of the wave paddles and the nonlinear propagation of the wave fronts. In this case, each wave was create using linear superposition of 244 wave fronts with frequencies evenly spaced between $0.101563 \mathrm{~Hz}$ and $2 \mathrm{~Hz}$. All waves in this study are non-breaking and trough focused, i.e. each of the contributing wave components has a phase of $\pi$ at a theoretical focus location, $x_{0}$. The amplitudes of the frequency components are derived by applying the NewWave theory (Tromans et al. 1991) to a JONSWAP spectrum with the parameters given in Table 1. Each wave front is then transformed back to the position of the wave paddles by the control software and $x_{0}$ is iteratively adjusted (as described by Hann et al. 2015) to pragmatically ensure focusing, i.e. a symmetric event, at the position coincident with the bow of the FPSO.

Wave parameters - Part 1

In Part 1, the three waves are all generated with an incident wave angle, $\alpha=0^{\circ}$. Two wave cases (11BT1 \& 12BT1) differ only by $H_{s}$ and so $12 \mathrm{BT} 1$ is essentially a steeper version of $11 \mathrm{BT} 1$ with the same relative frequency contributions; two cases (12BT1 \& 13BT1) differ only by peak frequency, $T_{p}$, so 13BT1 is essentially a steeper version of 12BT1 with the same $H_{s}$ (Table 1).

Wave parameters - Part 2

In Part 2, all three waves have the same steepness $(k A=0.17)$. The waves differ only by incident angle, $\alpha$ (Table 1).

Table 1 Wave conditions used in the CCP-WSI Blind Test Series 1

\begin{tabular}{llllll}
\hline & Case & $k A$ & $\alpha$ & $H_{s}$ & $T_{p}$ \\
\hline \hline \multirow{3}{*}{ Part 1 } & 11BT1 & 0.13 & $0^{\circ}$ & $0.077 \mathrm{~m}$ & $1.456 \mathrm{~s}$ \\
& 12BT1 & 0.18 & $0^{\circ}$ & $0.103 \mathrm{~m}$ & $1.456 \mathrm{~s}$ \\
& 13BT1 & 0.21 & $0^{\circ}$ & $0.103 \mathrm{~m}$ & $1.362 \mathrm{~s}$ \\
\hline \multirow{4}{*}{ Part 2 } & 21BT1 & 0.17 & $0^{\circ}$ & $0.103 \mathrm{~m}$ & $1.456 \mathrm{~s}$ \\
& 22BT1 & 0.17 & $10^{\circ}$ & $0.103 \mathrm{~m}$ & $1.456 \mathrm{~s}$ \\
& 23BT1 & 0.17 & $20^{\circ}$ & $0.103 \mathrm{~m}$ & $1.456 \mathrm{~s}$ \\
\hline
\end{tabular}

\section{Released Data}

The CCP-WSI Blind Test Series 1 is a blind validation of numerical WSI codes. Consequently the only physical measurement data released to participants prior to submission, were surface elevation data from the wave gauges in the empty tank tests (see Figures $3 \& 4$ a for the wave gauge positions). This data is deemed sufficient to reproduce the incident waves in each of the cases including the FPSO, for which the same wavemaker signals were used. The remaining physical measurements were not released until after all participants had submitted their final results and it is these 'blind' results that are reported in this paper.

\section{Physical Measurement Errors and Experimental Limitations}

As the predictive capability of the numerical submissions is judged on their ability to reproduce the physical results, it is absolutely necessary to also consider the errors present in the physical measurements. This is particularly pertinent in the cases presented here as, not only are the numerical results compared against the physical measurements but, all of the numerical simulations are initiated (in some way) using values derived from the physical measurements in the empty tank tests.

Typically the main source of random error, in experiments such as these, is due to reflected waves from the side walls and end of the wave basin. However, by utilising a compact wave group such as a focused wave the influence of reflected waves is less of an issue and occurs after the time of interest. Furthermore, limiting the wave cases to those without breaking greatly reduces the sources of random error associated with phenomena such as turbulence and aeration. Consequently the repeatability of the wave cases in this test is very good. In fact there is negligible difference in terms of phase between five repeated cases of the steepest wave. The only noticeable difference, between the repeats, is in the amplitude of the surface elevation at the time of focusing for which 
a relative standard deviation of $2.6 \%$ is observed. This is considered to be the maximum level of random error in the physical surface elevation measurements, in this test, and thus a maximum confidence interval of $\pm 7.8 \%$ in the run-up has been assumed, i.e. $99.7 \%$ of data values can be considered to be within three standard deviations.

Confidence in the pressure measurements is significantly lower. Due to the intermittent wetting of the pressure probes, some of the pressure records are effected by thermal shock. This results in nonphysical fluctuations in the pressure measurements at frequencies similar to those of the physical signal. Consequently, traditional filtering, based on frequencyspace methods, cannot remove this effect and so a bespoke 'thermal shock filter' has been designed to remove the unwanted signal. This new method is still under development and, as yet, is still not capable of distinguishing the smallest peaks in pressure from the background noise and has, therefore, resulted in these being removed from the filtered data. However, once applied, the pressure measurements from three experiments demonstrate excellent repeatability with the variation between test being lower than the noise in the signal $(\approx 0.005 \mathrm{~Pa})$.

Systematic errors in the physical measurements are considered to be negligible in the cases considered here. The wavelength of the waves is sufficient that minor inaccuracies in the probe positions (on the order of $10 \mathrm{~mm}$ ) are not important. The triggering of the various data acquisition systems is a potential source of issues. In these cases, because the numerical codes use the surface elevation measurements in the empty tank tests to initialise their models, the only issue is the triggering of the pressure probe measurements relative to the surface elevation measurements. The delay in the triggering is well understood in the COAST Laboratory and has been accounted for precisely in the results reported here.

\section{NUMERICAL METHODS}

\section{Participating Codes}

The CCP-WSI Blind Test Series 1 involved 33 participants, from 15 academic institutions, and industry collaborators, from 8 different countries. There are submissions using 10 different numerical codes/methods ranging from fully nonlinear potential theory (FNPT) to Navier-Stokes (NS) solvers; including hybrid (coupled) methods, partially particle methods, finite element methods, finite volume methods, both open-source and inhouse codes and both low-order accurate and high-order accurate codes. Each method is described below and the main characteristics of each implementation are summarised in Table 2.

\section{Particle-In-Cell (PIC) method}

This solver utilises the hybrid Eulerian-Lagrangian Particle-In-Cell (PIC) method to solve the incompressible NS equations for single-phase freesurface flows, incorporating a Cartesian cut cell-based, two-way, strong coupling algorithm for fluid-structure interaction (Chen et al., 2018; Chen \& Zang, 2018). In this method, the Lagrangian particles are used to solve the nonlinear advection terms and track the free-surface, while the Eulerian grid is employed for solving the non-advection terms with robustness and efficiency. The focused wave is generated using a pistontype wave paddle whose velocity and position are determined according to the NewWave theory and first-order wavemaker theory. Wave absorption is achieved through the relaxation zone approach. The computational domain is $24 \mathrm{~m}$ long, $3.025 \mathrm{~m}$ wide and $3.5 \mathrm{~m}$ high and is discretised by a uniform grid of size $0.025 \mathrm{~m}$ with eight particles being seeded in each cell accommodating the fluid area (which results in approximately 16 million grid cells and 105 million particles). The solution in time is first-order accurate and the time step is controlled by a Courant number of 0.5. No turbulence modelling is included, i.e. laminar flow is assumed. Computations are performed using 72 cores ( 6 Intel Xeon Gold 6126 CPUs) of an in-house high-performance computing (HPC) facility.
Table 2 Summary of numerical methods used by participants

\begin{tabular}{|c|c|c|c|c|}
\hline Code ref. & $\begin{array}{l}\text { Discret. } \\
\text { scheme }\end{array}$ & Theory & $\begin{array}{l}\text { Free-surface } \\
\text { treatment }\end{array}$ & $\begin{array}{l}\text { Turbulence } \\
\text { treatment }\end{array}$ \\
\hline PIC & $\begin{array}{l}\text { meshless } \\
+ \text { FVM }\end{array}$ & NS & MAC + & laminar \\
\hline $\begin{array}{l}\text { In-house NS } \\
1\end{array}$ & FVM & NS & VOF & $\begin{array}{l}\text { LES } \\
\text { (dynamic) }\end{array}$ \\
\hline $\begin{array}{l}\text { In-house NS } \\
2\end{array}$ & FVM & NS & VOF & laminar \\
\hline $\begin{array}{l}\text { FNPT } \\
(\text { FEM) }\end{array}$ & FEM & FNPT & single-phase & - \\
\hline Hybrid 1 & $\begin{array}{l}\text { FEM \& } \\
\text { FVM }\end{array}$ & $\begin{array}{l}\text { FNPT } \\
\& \text { NS }\end{array}$ & $\begin{array}{l}\text { single-phase } \\
\text { \& VOF }\end{array}$ & laminar \\
\hline $\begin{array}{l}\text { FNPT } \\
\text { (SEM) }\end{array}$ & SEM & FNPT & single-phase & - \\
\hline SWENSE & FVM & NS & level set & laminar \\
\hline $\begin{array}{l}\text { OpenFOAM } \\
\text { (IHFOAM) }\end{array}$ & FVM & NS & VOF & $\begin{array}{l}\text { RANS } \\
\text { (SST) }\end{array}$ \\
\hline Hybrid 2 & $\begin{array}{l}\text { Lagrangian } \\
\text { \& FVM }\end{array}$ & $\begin{array}{l}\text { Inviscid } \\
\& \text { NS }\end{array}$ & $\begin{array}{l}\text { Dynamic FS } \\
\text { \& VOF }\end{array}$ & laminar \\
\hline $\begin{array}{l}\text { OpenFOAM } \\
\text { (waves2Foam) }\end{array}$ & FVM & NS & VOF & laminar \\
\hline
\end{tabular}

\section{In-house NS solver 1}

This method utilises a two-phase flow model to solve the filtered NS equations using the finite volume method (FVM) and the high-resolution volume of fluid (VOF) scheme, CICSAM (compressive interface capturing scheme for arbitrary meshes), on a staggered Cartesian grid. The partial cell treatment in 3D is used to allow for complex geometries in the domain (Xie, 2015). The advection terms are discretised by a highresolution scheme which combines the high order accuracy with monotonicity (Xie, 2012). The gradients in pressure and diffusion terms are obtained by central difference schemes. An expression-based boundary condition, based on the superposition of linear wave components derived from the empty tank tests, is used to define the time history of wave elevations and velocities at the inlet. For wave absoption, a radiation outlet boundary condition is used. In this study, the computational domain is $6 \mathrm{~m}$ long, $3 \mathrm{~m}$ wide and $3.3 \mathrm{~m}$ high; it has a uniform mesh with size $0.01875 \mathrm{~m}$ in the horizontal and $0.01 \mathrm{~m}$ in the vertical (total number of mesh cells $\approx 16.4$ million). The SIMPLE algorithm for the pressurevelocity coupling is employed and a backward finite difference discretisation is used for the time derivative (Xie et al., 2018). Large-eddy simulation is employed for the turbulence modelling with the dynamic Smagorinsky model. The computation of the test cases was performed using $512 \times 2.6 \mathrm{GHz}$ cores of an in-house HPC cluster.

\section{In-house NS solver 2}

naoe-FOAM-SJTU is an in-house solver based on OpenFOAM's default interDyMFoam, which solves the Reynolds-Averaged Navier-Stokes (RANS) equations for two, incompressible, isothermal and immiscible fluids using a VOF interface capturing scheme, with additional wave generation and absorption capabilities (Shen et al., 2014). Wave generation is achieved using expression based boundary conditions based on linear superposition of wave components derived from the same theoretical wave conditions as those used in the physical experiment. Wave absorp- 
tion is achieved via an artificial viscous term in the momentum equation, i.e. a sponge layer. In this study, the computational domain is $23 \mathrm{~m}$ long and $4 \mathrm{~m}$ wide. The mesh is unstructured with a free-surface refinement layer consisting of $>40$ cells over the wave height and $>80$ cells over the characteristic wavelength; further refinement is present around the FPSO ( $\approx 5.7$ million cells in total). A fixed time step of $d t=0.01 \mathrm{~s}$ is used throughout. No turbulence modelling is included, i.e. laminar flow is assumed. The computation of the test cases was performed using $64 \times$ $2.8 \mathrm{GHz}$ cores (IBM NeXtScale nx360 m4 model) (Li et al., 2018a).

\section{FNPT solver (using FEM)}

In QALE-FEM (Quasi Arbitrary Lagrangian-Eulerian Finite Element Method) the flow is governed by the FNPT model where a boundary value problem for velocity potential is solved using the FEM. A similar boundary value problem is solved to find the time derivative of the velocity potential in the Bernoulli equation, which is used to find the force acting on floating structures. The fully nonlinear free surface conditions are written in arbitrary Lagrangian-Eulerian forms (Ma \& Yan, 2006; Yan \& Ma 2010). Wave generation and absorption is achieved using self-adaptive wavemakers. In this study, as the incident waves are uni-directional, a quasi-2D domain is combined with a 3D domain using a weak zonal coupling. The $2 \mathrm{D}$ domain has the same dimensions as the experimental wave basin (Figure 2) but is very coarse in the direction normal to the wave propagation, i.e. 5 cells wide, whereas the $3 \mathrm{D}$ domain consists of a cylindrical domain (radius $3.5 \mathrm{~m}$ ) centered on the FPSO. The 3D domain has a graded mesh with a characteristic mesh size of $0.03 \mathrm{~m}$ near the free-surface and $0.02 \mathrm{~m}$ on the surface of the FPSO ( $\approx 3.45$ million mesh cells total). The time discretisation scheme is a 2nd order finite difference scheme and the time step is fixed at $128 \mathrm{~Hz}$ to be consistent with the physical data. The computation of the test cases was performed using $4 \times 2.9 \mathrm{GHz}$ cores (Xie et al., 2018).

\section{Hybrid method 1}

The hybrid FNPT-NS solver, qaleFOAM, combines QALE-FEM (see above) and OpenFOAM's multiphase NS solver interDyMFoam using a domain decomposition method and a coupling boundary ( $\mathrm{Li}$ et al. 2018b). Wave generation and absorption is achieved using self-adaptive wavemakers on the inlet and outlet of the the FNPT region which covers the whole computational domain with the same size of the experimental wave basin (Figure 2). The mesh in the FNPT domain has a characteristic size of $0.04 \mathrm{~m}$. The NS domain is confined to a small region $(5.4 \mathrm{~m} \times 3 \times$ $3.53 \mathrm{~m}$ ) surrounding the structure and is bounded by the coupling boundary upon which the velocity, pressure and wave elevation values for the NS solver are provided by the QALE-FEM. The mesh in the NS region is graded with approximately 50 cells over the maximum wave height and 180 cells per peak wavelength in the free-surface region and a characteristic cell size of $0.005 \mathrm{~m}$ on the FPSO surface $(\approx 1.96$ million mesh cells total). The time stepping is first order accurate (Implicit Euler) and dynamic, based on a Courant-Friedrichs-Lewy (CFL) condition of 0.5. No turbulence modelling is included, i.e. laminar flow is assumed. The computation of the test cases was performed using $8 \times 2.6 \mathrm{GHz}$ cores.

\section{FNPT solver (using spectral element method (SEM))}

This method is based on a stabilised spectral element method (SEM) using a Galerkin spatial discretization and an explicit Runge-Kutta method for the temporal integration of an Eulerian formulation of the fully nonlinear potential flow (FNPF) equations (Engsig-Karup et al., 2016; Engsig-Karup \& Eskilsson, 2018). The FNPF-SEM solver is high-order accurate and represents the solution variables on an unstructured mesh through the globally continuous piece-wise polynomial basis functions. Wave generation and wave absorption is done using a standard embedded penalty method (Engsig-Karup et al., 2013). Incident waves are gener- ated using superposition of first-order linear wave components derived from the empty tank data using a discrete Fourier transform. The computation domain is $12 \mathrm{~m}$ long and $8 \mathrm{~m}$ wide. The grid is relatively coarse consisting of two vertical layers and a total number of prism elements on the order of $10,000(\approx 500,000$ nodes (degrees of freedom) in the fluid volume discretisation). The FPSO is captured to high accuracy using curvilinear faces in the top layer that also account for the curvilinear free surface representation. The time-stepping is based on a fourth-order explicit Runge-Kutta method with time step sizes of $d t=0.025 \mathrm{~s}$ in all simulations. The computations of the test cases were performed in MATLAB R2017a and executed sequentially (i.e. no code parallelisation).

\section{Spectral wave explicit Navier-Stokes equations (SWENSE) solver}

The Naval Hydro Pack is a specialised software library based on the foam-extend, collocated finite volume computational fluid dynamics (CFD) software. In this method the spectral wave explicit Navier-Stokes equations (SWENSE) method is used to couple the incident wave field and the CFD solution (Vukĉević et al., 2016). A two-phase flow model is used with the level set method for interface capturing; the ghost fluid method is used to take into account the discontinuities in fields across the interface (Vukĉević et al., 2017). Waves are generated using implicit relaxation zones (Jasak et al., 2015) which are positioned along the edge of the computational domain which, in the cases studied here, is $20.7 \mathrm{~m}$ long, $7 \mathrm{~m}$ wide and $3.53 \mathrm{~m}$ high. The mesh is graded towards the structure, with a resolution corresponding to $\Delta x=0.01 \mathrm{~m}, \Delta y=0.02 \mathrm{~m}$ and $\Delta z=0.005 \mathrm{~m}$ in the vicinity of the hull surface, resulting in approximately 4.1 million cells in total. The time-step is controlled to maintain a CFL number below 1, resulting in time-steps ranging from 0.005 to $0.015 \mathrm{~s}$. An implicit second order backward time marching scheme is used. No turbulence models are used, instead laminar flow is simulated. Simulations are performed using 21 cores of the Intel Xeon Processor E5-2637 v3 (15M Cache, $3.50 \mathrm{GHz}$ ) (Gatin et al., 2018).

\section{Open-source NS solver (OpenFOAM using IHFOAM)}

This method utilises OpenFOAM (ESI version 1706), an open-source CFD software based on the finite volume discretisation. The standard interFoam solver is used to solve the RANS equations for two, incompressible, isothermal and immiscible fluids using a VOF interface capturing scheme. The IHFOAM toolbox provides the functionality of wave generation and active wave absorption (Higuera et al., 2013). A member function has been added to generate phase-focused waves based on second order irregular wave theory and wave components derived from the given wave conditions and spectra. The computational domain is $10 \mathrm{~m}$ long, $4 \mathrm{~m}$ wide and $3.43 \mathrm{~m}$ high. The spatial discretisation takes the form of an unstructured mesh, consisting mostly of hexes, with a typical resolution of $0.0085 \mathrm{~m}$ in the region of the free surface, and $0.00425 \mathrm{~m}$ around the structure. The total number of mesh cells used in each case is approximately 3.1 million. The time stepping is first order accurate (Implicit Euler) and dynamic, based on a CFL condition of 0.35 . No turbulence modelling is included, i.e. laminar flow is assumed. The computations were performed using $48 \times 1.7 \mathrm{GHz}$ cores of an in-house HPC facility.

\section{Hybrid method 2}

This hybrid method utilises a Lagrangian wave model (Buldakov, 2013) to provide the inlet boundary conditions for the two phase, incompressible, RANS solver, olaFlow (developed in the OpenFOAM framework) (Higuera, 2017). The Lagrangian model reproduces the shape of the physical wave basin (Figure 2) in two dimensions and, utilising the iterative methodology of Buldakov et. al. (2017), reconstructs the flap wavemaker motion required to generate the experimental surface elevation signal (6 to 7 iterations were required to achieve the desired convergence level). Wave kinematics corresponding to the final iteration are 
used as inlet boundary conditions in the RANS simulations. To ensure the target waves are generated regardless of any reflected waves reaching the boundary a new one-way coupling technique has been developed (Higuera et al., 2018). Incident waves are also absorbed at the opposite boundary using the same technique coupled with an enhanced version of the active wave absorption presented in Higuera et al. (2013). The spatial discretisation in the Lagrangian model is $251 \times 16$ and the time step is $0.005 \mathrm{~s}$. The CFD mesh is $15 \mathrm{~m} \times 1.7 \mathrm{~m} \times 3.16 \mathrm{~m}$ and unstructured, but hexahedral-cell dominant, with a maximum cell resolution of $0.01 \mathrm{~m}$, adjacent to the FPSO (total number of cells $\approx 46.5$ million). The time stepping is first order accurate (Implicit Euler) and dynamic, based on a CFL condition of 0.15 . No turbulence modelling is included. The computation of the test cases was performed using $120 \times 2.6 \mathrm{GHz}$ cores.

\section{Open-source NS solver (OpenFOAM using waves2Foam)}

This method utilises OpenFOAM (Foundation version 4.1), an opensource CFD software based on the finite volume discretisation (Brown et al. 2018). A modified version of the standard interFoam solver is used to solve the RANS equations for two incompressible, isothermal and immiscible fluids using a VOF interface capturing scheme. The waves2Foam toolbox (Jacobsen et al., 2012) provides wave generation through a linear superposition of first order wave components (hierarchically selected based on a fast Fourier transform (FFT) of the physical empty tank data (Musiedlak et al. 2017)). Wave absorption is achieved using the relaxation zone functionality provided as part of the waves 2 Foam toolbox. The computational domain is $14 \mathrm{~m}$ long, $6 \mathrm{~m}$ wide and $4 \mathrm{~m}$ high. The spatial discretisation takes the form of an unstructured mesh, consisting mostly of hexes, with a typical resolution of $0.025 \mathrm{~m}$ in the region of the free surface, and $0.00625 \mathrm{~m}$ around the structure. The total number of mesh cells used in each case is approximately 1.9 million. The time stepping is first order accurate (Implicit Euler) and dynamic, based on a CFL condition of 0.25 . No turbulence modelling is included, i.e. laminar flow is assumed. The computation of the test cases was performed using $16 \times 2.6 \mathrm{GHz}$ cores of an in-house HPC facility.

\section{Submissions}

Participation in the CCP-WSI Blind Test Series 1 is purely voluntary and so, as a consequence, some participants only managed to complete a selection of the test cases. Table 3 summarises the test cases that were submitted for each code/method (where an ' $X$ ' signifies a submission).

\section{RESULTS \& DISCUSSION}

\section{Time Series Analysis}

As highlighted in Figures 3, 4 and 5, participants were requested, in each of the six cases, to submit times series data for five different surface elevation probe positions and six different pressure probe positions. As a result, a huge data set is available for the comparative study. However, the processing of all the data has been deemed impractical and so, after reviewing all of the data qualitatively, a representative sample has been selected and reported here. In general, the same trends are observed for all of the measurements and so the run-up on the bow (probe WG16) and the pressure on the bow, at the still water level (probe P2), have been considered as being arguably most relevant to design engineers etc.

\section{Run-up at the bow}

Figure 6 shows the physical measurements (including the $99.7 \%$ confidence interval), as well as all of the numerical submissions, for run-up on the bow of the FPSO, for all three cases in Part 1, i.e. for increasingly steep waves with zero incident wave angle. The results show that, for the range of wave steepness considered, all of the numerical methods
Table 3 Submitted test cases for each of the participating codes

\begin{tabular}{lcccccc}
\hline Code ref. & 11BT1 & 12BT1 & 13BT1 & 21BT1 & 22BT1 & 23BT1 \\
\hline \hline PIC & $\times$ & $\times$ & - & $\times$ & $\times$ & $\times$ \\
\hline $\begin{array}{l}\text { In-house NS } \\
1\end{array}$ & - & $\times$ & - & - & - & - \\
\hline $\begin{array}{l}\text { In-house NS } \\
2\end{array}$ & $\times$ & $\times$ & $\times$ & - & - & - \\
\hline $\begin{array}{l}\text { FNPT } \\
\text { (FEM) }\end{array}$ & $\times$ & $\times$ & $\times$ & $\times$ & $\times$ & $\times$ \\
\hline Hybrid 1 & $\times$ & $\times$ & $\times$ & - & - & - \\
\hline $\begin{array}{l}\text { FNPT } \\
(\text { SEM) }\end{array}$ & $\times$ & $\times$ & $\times$ & $\times$ & $\times$ & $\times$ \\
\hline SWENSE & $\times$ & $\times$ & $\times$ & $\times$ & $\times$ & $\times$ \\
\hline $\begin{array}{l}\text { OpenFOAM } \\
\text { (IHFOAM) }\end{array}$ & $\times$ & $\times$ & $\times$ & - & - & - \\
\hline Hybrid 2 & - & $\times$ & $\times$ & - & - & - \\
\hline $\begin{array}{l}\text { OpenFOAM } \\
(\text { waves2Foam) }\end{array}$ & $\times$ & $\times$ & $\times$ & $\times$ & $\times$ & $\times$ \\
\hline
\end{tabular}

are able to predict the general behaviour well, particularly the phasing of the wave event, and that there is no obvious trend between the predictive capability of the methods and their underlying complexity, i.e. for the (non-breaking) cases consider here, there appears to be no clear advantage in using high fidelity methods over FNPT. There is, however, quite a range in the predicted amplitudes of the crests and troughs with a distinct tendency for the numerical simulations to over-predict, particularly the crest immediately following the main trough. Qualitatively, it is possible that these discrepancies increase with wave steepness, i.e there appears to be significant difficultly in reproducing the crest immediately before the main trough in the steepest case (Figure 6c). However, quantitative analysis is required to provide conclusive evidence of this (and there is still no evidence that higher-fidelity models perform better, even for the steepest case considered here). Lastly, a few seconds after the main trough, the predictive capability of the numerical models is greatly reduced and the variation in the predictions is large. However, this observation is almost certainly due to the differing treatment of reflected waves in each of the numerical methods; as the reflective characteristics of the physical beach were not released, participants are not expected to be able to reproduce this part of the time series nor is it used to judge the submissions.

Figure 7 shows the run-up on the front of the FPSO, for all three cases in Part 2, i.e. for a fixed wave steepness and increasing incident wave angle. The same observations can be made as above: all numerical methods perform reasonably well with well-reproduced phasing in each of the cases; there is some variation in the amplitudes with a tendency for overprediction, but again; there is no obvious advantage in using high-fidelity methods for the cases considered in this study. Lastly, in the cases considered here, when considering the run-up on the bow of the FPSO, there does not appear to be any trend between the predictive capability of the numerical models and the incident wave angle.

\section{Pressure on the bow}

Figures 8 and 9 show the physical measurements, as well as all of the numerical submissions, for pressure on the bow of the FPSO at the still water level (SWL), for all three cases in Part 1 and Part 2 respectively. The pressure results show similar trends to the run-up: all submissions repro- 

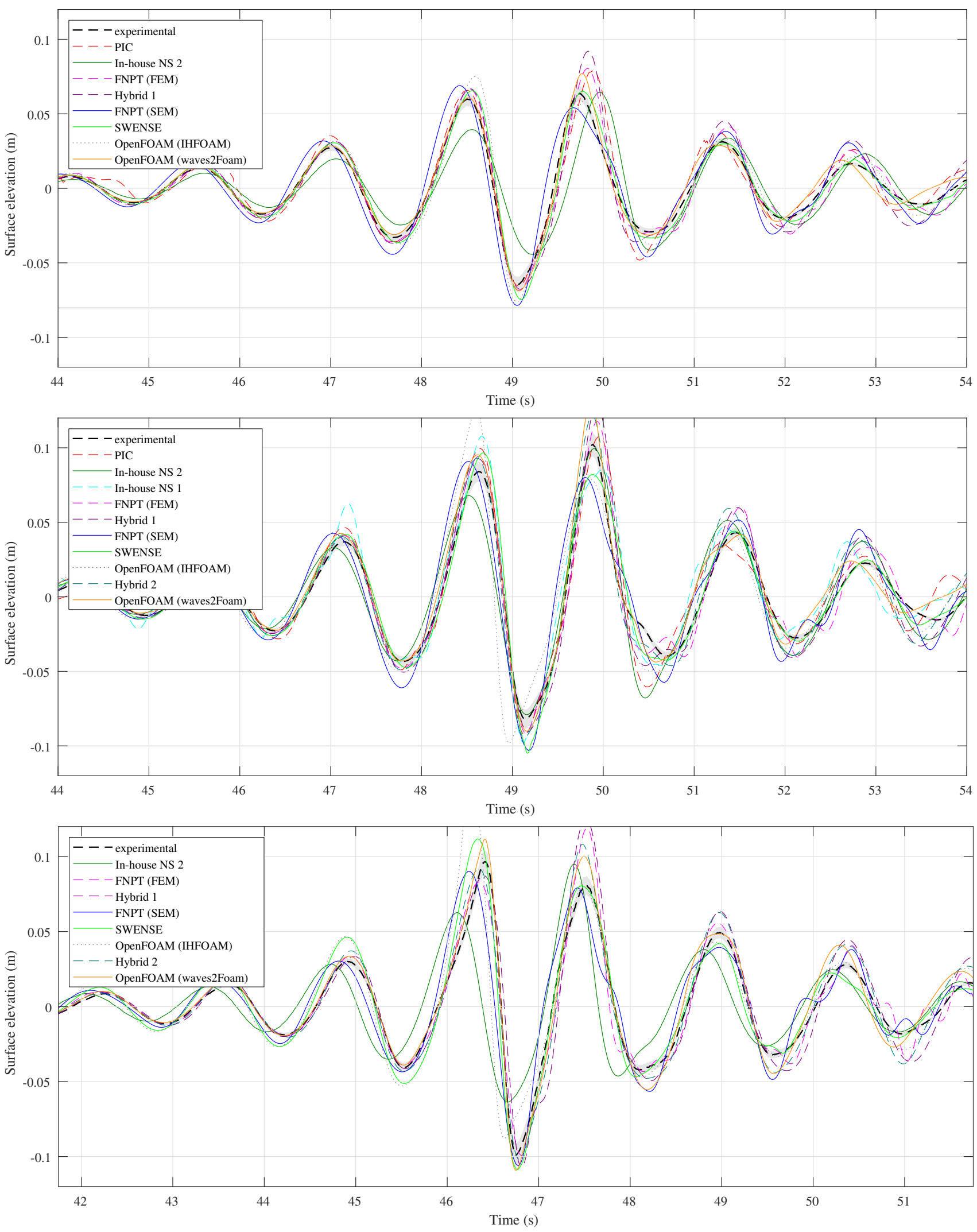

Fig. 6 Run-up on the bow of the FPSO for cases in Part 1 (Probe WG16), i.e. increasing in steepness from (a) $11 \mathrm{BT} 1(k A=0.13)$ to (b) $12 \mathrm{BT} 1$ $(k A=0.18)$ to $(\mathrm{c}) 13 \mathrm{BT} 1(k A=0.21)$. Physical measurements are plotted using a black dotted line (including the $99.7 \%$ confidence interval shaded in grey). Numerical submissions, from all participants, are shown using solid lines. 

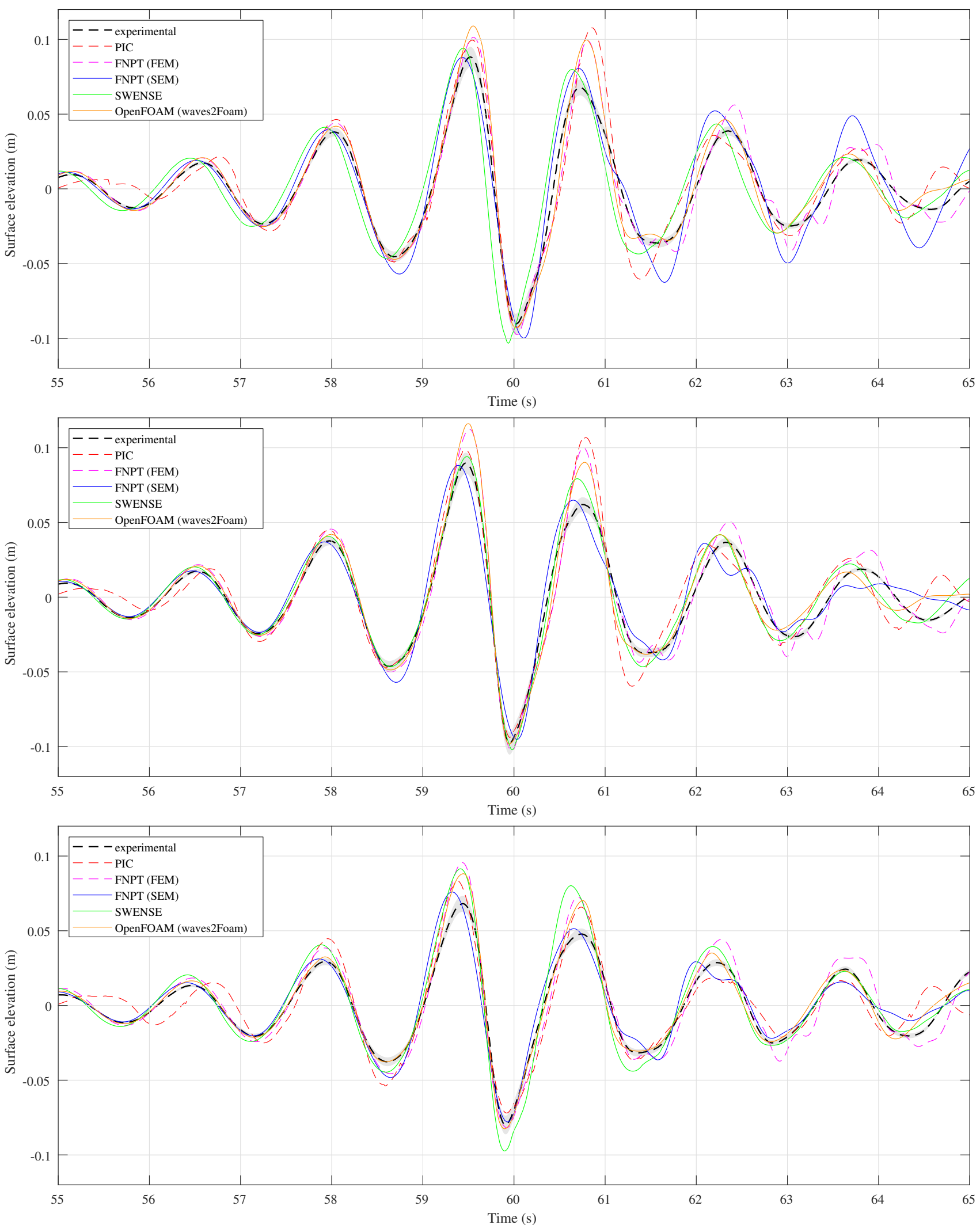

Fig. 7 Run-up on the bow of the FPSO for cases in Part 2 (Probe WG16), i.e. increasing angle of incidence from (a) $21 \mathrm{BT} 1\left(\alpha=0^{\circ}\right)$ to (b) $22 \mathrm{BT} 1$ $\left(\alpha=10^{\circ}\right)$ to (c) 23BT1 $\left(\alpha=20^{\circ}\right)$. Physical measurements are plotted using a black dotted line (including the $99.7 \%$ confidence interval shaded in grey). Numerical submissions, from all participants, are shown using solid lines. 

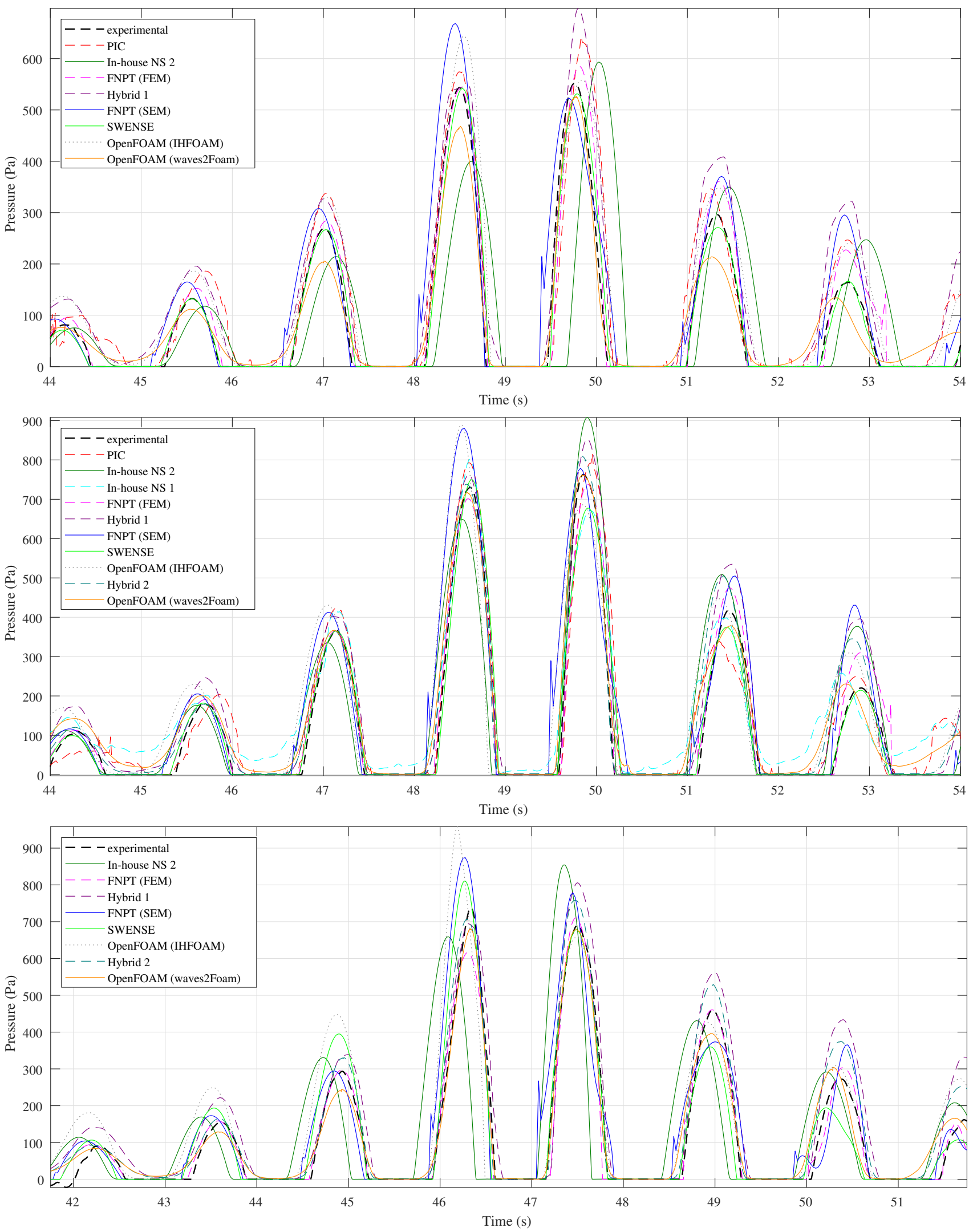

Fig. 8 Pressure on the bow of the FPSO, at the still water level (probe P2), for cases in Part 1, i.e. increasing in steepness from (a) 11BT1 $(k A=0.13)$ to (b) 12BT1 $(k A=0.18)$ to (c) 13BT1 $(k A=0.21)$. Physical measurements are plotted using a black dotted line. Numerical submissions, from all participants, are shown using solid lines. 

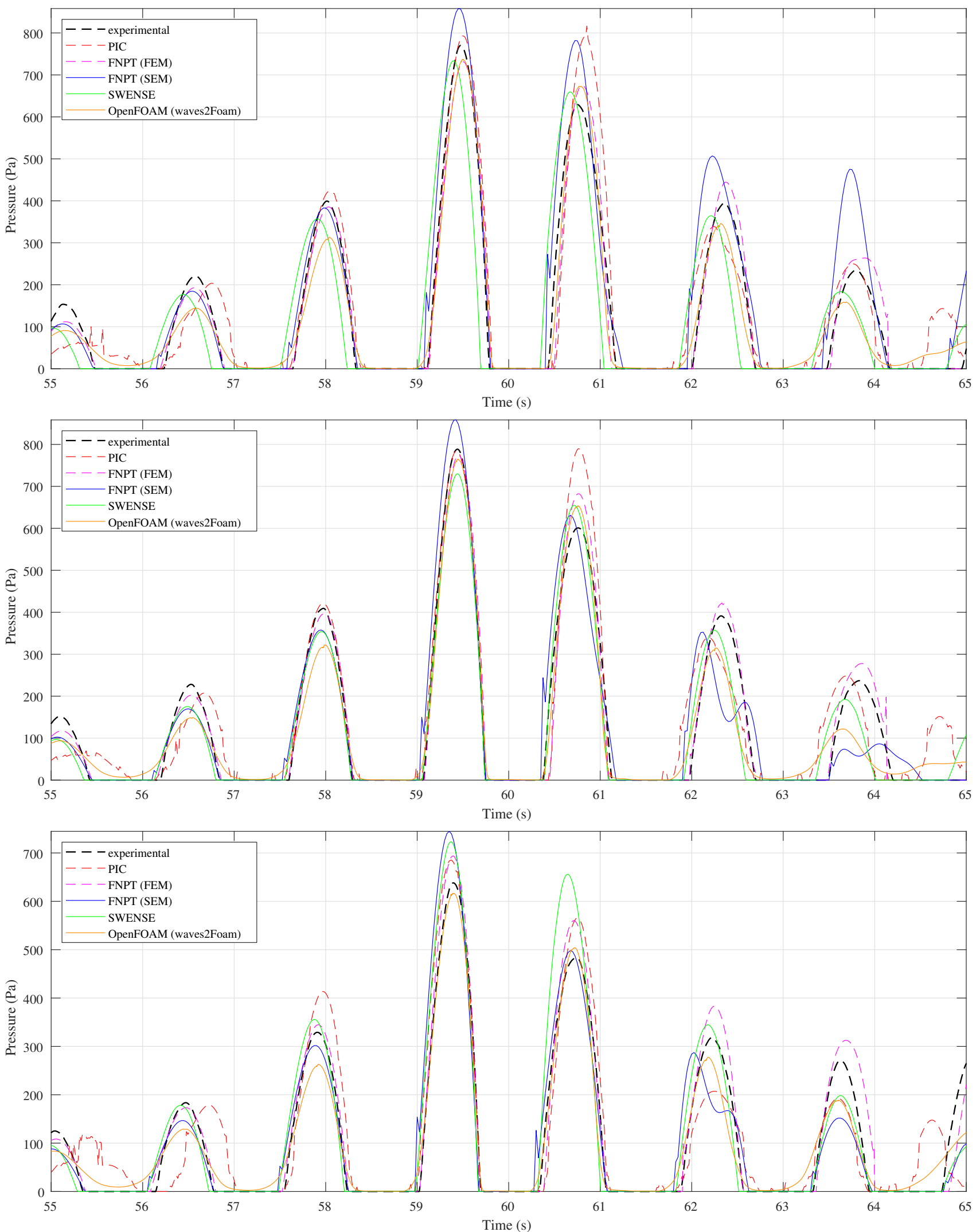

Fig. 9 Pressure on the bow of the FPSO, at the still water level (probe P2), for cases in Part 2, i.e. increasing angle of incidence from (a) 21BT1 $\left(\alpha=0^{\circ}\right)$ to (b) 22BT1 $\left(\alpha=10^{\circ}\right)$ to (c) 23BT1 $\left(\alpha=20^{\circ}\right)$. Physical measurements are plotted using a black dotted line. Numerical submissions, from all participants, are shown using solid lines. 
duce the pressure reasonably well, there appears to be a greater spread in the predicted phasing compared to the surface elevation, and there is considerable variation in the predicted amplitude of the peaks (again typically over-estimates). Again, the predictive capability of the numerical models is greatly reduced a few seconds after the main peaks but this can again be attributed to the variation in the treatment of reflected waves, but, there is again no clear relationship between the predictive capability of the models and either the incident wave angle or the wave steepness. However, in the steepest case (Figure 8c), the predictions of the crest immediately before the main trough do appear to show the greatest variability. There is, again, no obvious benefit in using higher-fidelity methods for the (non-violent) wave cases considered here. Lastly, it has been noticed that there is a large variation in the prediction of hydrostatic pressure between the numerical methods.

\section{Quantitative Analysis}

Comparisons of time series' are valuable, in that all the data are present without manipulation or the introduction of bias (from analysis methods that favour one data set over another). However, only qualitative comparisons can be drawn directly and with so many participants and with multiple cases to analyse, it is not possible to ascertain conclusive evidence of any parametric trends in the data. Therefore, a series of strategies are investigated here in order to quantify the predictive capability of the numerical methods into a limited number of discrete values and allow for more simplified visualisation of any trends in the data.

\section{Root Mean Squared (RMS) error}

A root mean squared (RMS) error calculation is perhaps the simplest estimation of the 'error' associated with any particular reproduction of a time series. An RMS error, in these cases, is defined as the square root of the arithmetic mean of the squares of the differences between the reproduction (numerical submission) and the original (the physical data). This then returns a single number representation of the predictive capability of the numerical method. However, in the general case, the time vector of the original time series does not match that of the reproduction (particularly when the numerical method uses adaptive time-stepping) and so some form of interpolation is typically required before an RMS can be calculated, adding an additional layer of uncertainty around the prediction. Furthermore, although convenient for analysis of many multivariant data sets, reducing the measure of predictive capability to a single number removes large amounts of (potentially valuable) information about the reproduction and can lead to a significant bias in the results. For example, in an otherwise perfect reproduction of the original time series, a small phase shift in the prediction can result in a very large RMS error, particularly in cases with steep gradients in the measured quantity, and so this form of analysis favours those methods that predict the phases well. Therefore, in cases in which the timings of events are less critical (compared to the amplitude), an RMS is not an appropriate measure of the quality of the prediction. Despite this, the RMS in the predicted runup on the bow of the FPSO has been calculated (for a window of time starting 5 seconds before the main trough in the physical data and ending 5 seconds after) and plotted against both the waves steepness (Figure 10) and the incident wave angle (Figure 11). Also plotted is the RMS error in each submission against the CPU effort for each simulation (Figure 12). CPU effort has been estimated as the execution time, multiplied by the number of cores used, divided by the simulated time (it should be noted that this estimation does not account for the different hardware used by each of the participants, nor were participants asked to minimise the CPU effort in their submissions). In each figure, the data has been colour-coded according to the underlying theory/method, i.e. red symbols symbolise NS solvers, blue symbolises FNPT solvers, cyan is used for the hybrid methods and the PIC method is coloured in magenta.

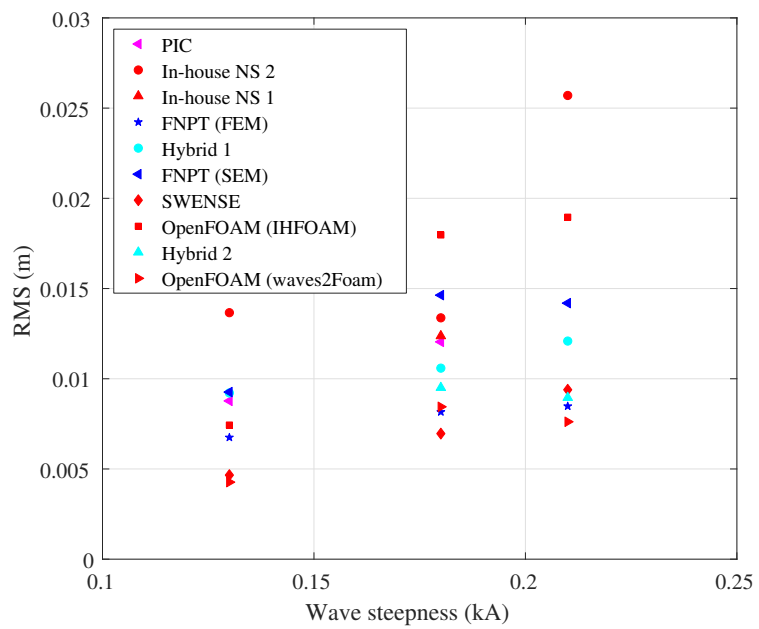

Fig. 10 RMS vs. wave steepness for numerical predictions of runup on the bow of the FPSO (WG16), in Part 1.

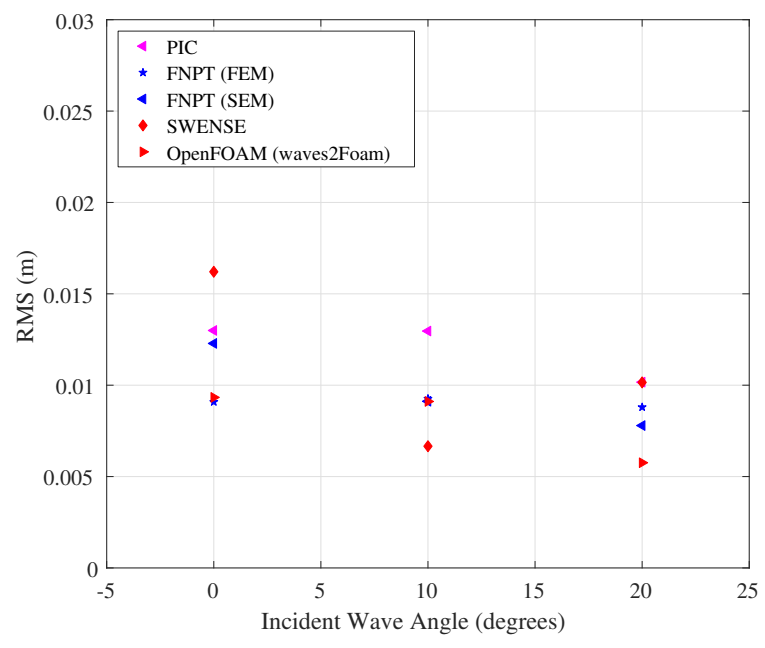

Fig. 11 RMS vs. incident wave angle for numerical predictions of run-up on the bow of the FPSO (WG16), in Part 2.

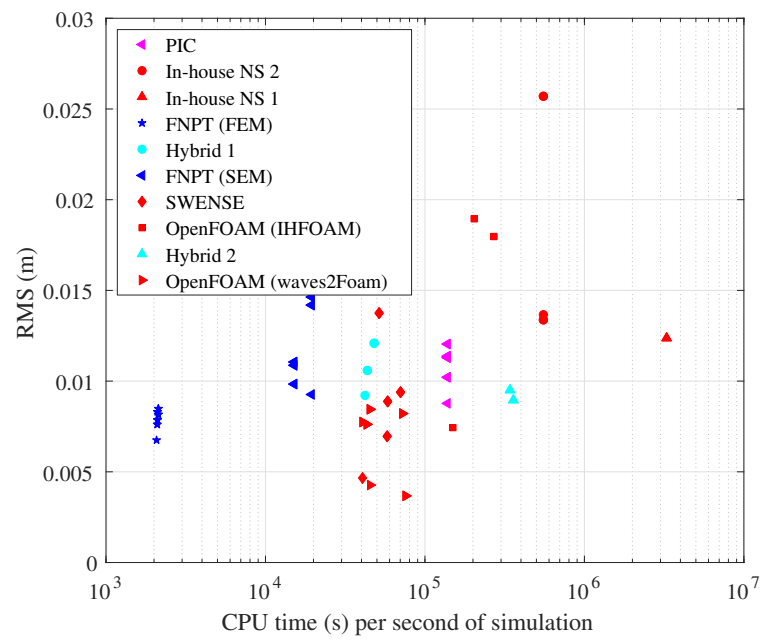

Fig. 12 RMS vs. execution time for numerical predictions of runup on the bow of the FPSO (WG16), (both Parts $1 \& 2$ ). 
There is significant scatter in the RMS values calculated and so low confidence in any trends observed. There appears to be a reduction in the RMS as the incident wave angle increases and potentially an increase in the RMS as the wave steepness increase. However, the amplitude of the run-up on the centre of the bow is also seen to reduce with incident wave angle and the most significant increase in RMS occurs between cases 11BT1 and 12BT1 $(0.13<k A<0.18)$, i.e. a rise in steepness due to an increase in wave amplitude. Therefore, it is suspected that any increase in RMS is likely due to an increase in wave amplitude and that steepness and direction does not have a dominant effect in these cases. Furthermore, as observed in the qualitative analysis of the time series data, the FNPT methods have performed equally as well as the high fidelity methods; it could be argued that the NS solvers achieve the lowest RMS values but NS solvers are also responsible for the greatest RMS values highlighting a potential concern over the apparently high variability in the results produced when solving fundamentally the same set of equations. Figure 12 shows, perhaps, the most conclusive result; FNPT solvers are significantly quicker at solving these cases than NS solvers without a significant decrease in the RMS error. The FEM-based FNPT method is at least 1.5 orders of magnitude faster than the quickest NS code and has comparable predictive capability in these cases. The FNPT method based on SEM is significantly slower in comparison and has a slightly greater RMS, however, this is a relatively new implementation of the method and with further refinement it is anticipated that this method will be competitive with the more established FNPT (FEM) method. Again, NS solvers display both the lowest and highest RMS values and the spread in CPU effort for NS solvers is also very large. It is suspected that these variations are due mostly to the wide range of mesh/domain designs, wave generation/absorption strategies and CPU resources used. This highlights that, either 'best practice' procedures for these applications are not known or participants are not adhering strictly to them. Finally, the two hybrid methods, although relatively new implementations, do not display any benefit over the other methods, i.e. in the cases reported here, there is no saving of CPU effort over NS-only solvers nor is there any improvement in the prediction over FNPT methods.

\section{Multi-value assessment methods}

As mentioned above, although convenient for identifying trends across multiple cases, the RMS error may not be an appropriate measure of the predictive capability of a code, particularly in some cases where significant bias is introduced, i.e. cases with high gradients and small phase discrepancies. Furthermore, as a single-value representation of accuracy, the RMS does not allow for differentiation between the errors associated with individual variables in multivariant data sets, i.e. amplitude, phase, frequency-content etc. In fact it is not possible for a single-value assessment method to provide this information directly; the method must produce at least the same number of values as the variables under scrutiny.

A number of multi-value assessment methods have been considered here: The cross-correlation can be used to find the similarity between two time series as a function a time shift (phase shift) of one series relative to the other; the 'lag' associated with the maximum similarity and the value of the maximum similarity then gives a two-value estimate of the reproduction incorporating a measure of the phase prediction and the amplitude prediction without introducing a bias due to a phase discrepancy (this is essentially equivalent to shifting the predicted time series in time to minimise the RMS error and then recording the time shift and the minmised RMS. This idea is also equivalent to performing the linear least-squares approach from regression analysis which minimises the sum of the squares of the differences between the physical value and the model data points). This method, however, does not provide any information regarding the frequency content in the reproduction and a poor similarity could be due to either a frequency content discrepancy or an amplitude discrepancy. An alternative assessment method could be to use a dynamic time warping (DTW) algorithm which determines a measure of similarity independent of nonlinear variations in the time dimension, i.e. unlike the cross-correlation, the sequence alignment method, or 'warping', is non-uniform and nonlinear. DTW returns a 'distance-like' quantity, know as the Euclidean distance, describing the 'distance' between the two signals in the time dimension which incorporates discrepancies in both phase and frequency content. A two-value assessment can then be completed by performing an RMS analysis of the target and the warped time series' giving an error estimate in the amplitude that is arguably independent of any inaccuracy in the phase or frequency content. Frequency domain analysis methods, such as Fourier transforms, offer a multi-valued assessment method consisting of the power (amplitude) as a function of frequency. An FFT can also allow for phase comparisons as a function of frequency. Windowed frequency domain analysis methods, such as Bartlett's or Welch's method, as well as wavelet transformations also return the power as a function of frequency with the additional benefit of providing the assessment over a finite time (and so a series of assessments in time). These methods can be beneficial for understanding the temporal variation in the quality of the reproduction. Further strategies, such as time series of cumulative residuals between the physical and numerical data, can give the relative error with respect to time and can provide insight into the reproduction as a function of time.

A problem arises, however, when interpreting the results from these various analysis methods; the more complex methods in particular tend to generate comparable size data sets to the original time series and when it comes to comparing multiple participants data across multiple cases, i.e. introducing further variables, the interpretation of this data becomes far from trivial. Without further processing of the output to reduce the number of assessment values it is not even possible to represent the data in a meaningful way for the benefit of interpretation. Therefore, some compromise is required over the level of reduction obtained via the assessment methodology and the detail left to make the assessment. A single-value assessment method, like the RMS, is preferable as many cases covering a key variable such as incident wave steepness can be represented on a simple $2 \mathrm{D}$ plot and a trend can then be quantified easily. Two-value assessment methods may be optimal as, by utilising a 3D plot, the two assessment variables can both be represented, along with the key case variable, and trends observed/quantified easily. However, these methods both result in a drastic reduction in the information used to make the assessment and are susceptible to bias as a consequence.

This dilemma highlights possibly the most important question when designing a comparative study, i.e which variable, or variables, are most crucial in the assessment of predictive capability, and, in the case of multiple variables how should each of them be weighted in terms of importance? If this were well defined, perhaps appropriate assessment strategies could be defined and a weighted sum of the errors could be used to give a single value for the predictive capability. This could then be described as a function of key case parameters and a parametric understanding of the practical applicability of participating methods be formed.

\section{Discrete value assessment}

It is common, in the practical application of numerical models, that assessment of the predictive capability over a certain period of time is not necessary and that critical design parameters are based on single discrete values such as maxima in the load or run-up. For example, in the context of extreme wave events interacting with an FPSO, design engineers may only be interested in the maximum pressure or the maximum run-up on the bow to ensure structural integrity and account for any green-water effects respectively. Provided the key assessment values are well conceived, this kind of reduction in the data greatly reduces the complexity of any comparative study and parametric trends based on key variables 


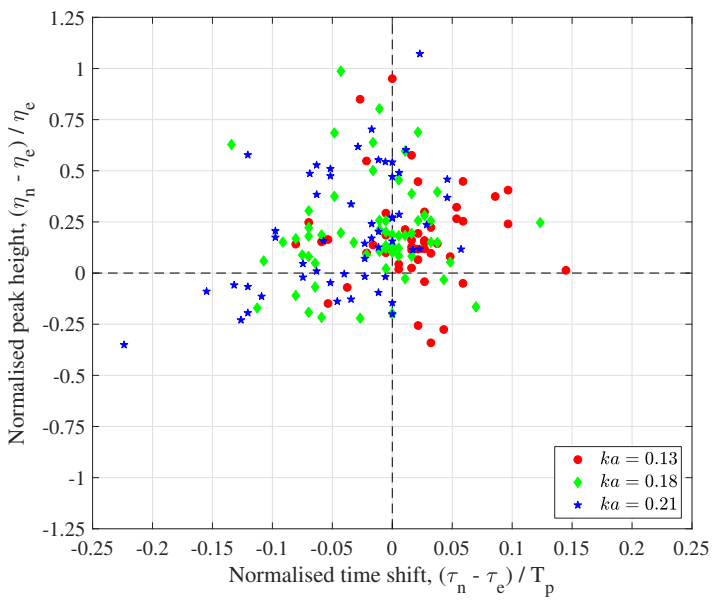

Fig. 13 Normalised peak height vs. normalised time shift for peaks in run-up on the bow of the FPSO, for all participants and cases in Part 1 . Colour coded by wave steepness.

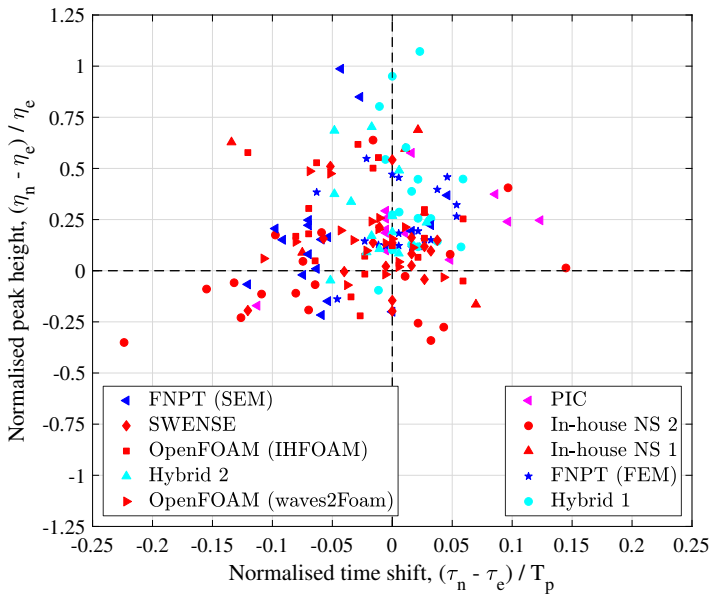

Fig. 14 Normalised peak height vs. normalised time shift for peaks in run-up on the bow of the FPSO, for all participants and cases in Part 1. Colour coded by code type.

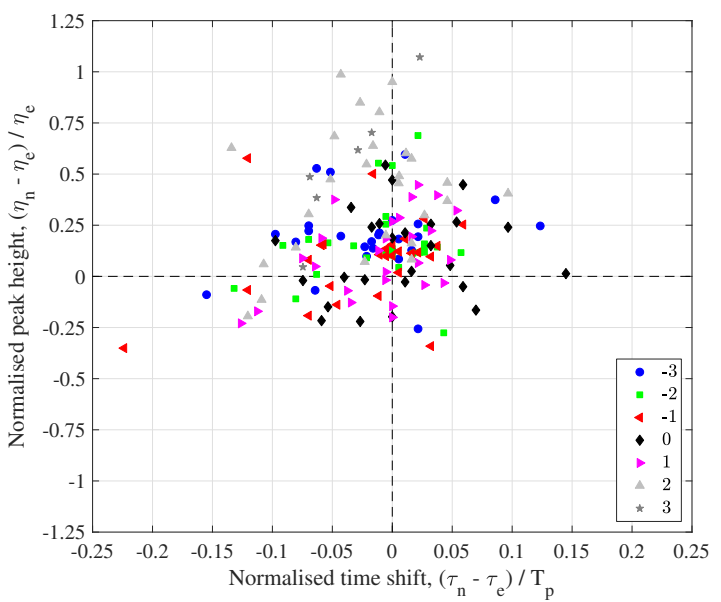

Fig. 15 Normalised peak height vs. normalised time shift for peaks in run-up on the bow of the FPSO, for all participants and cases in Part 1. Colour coded by peak number.

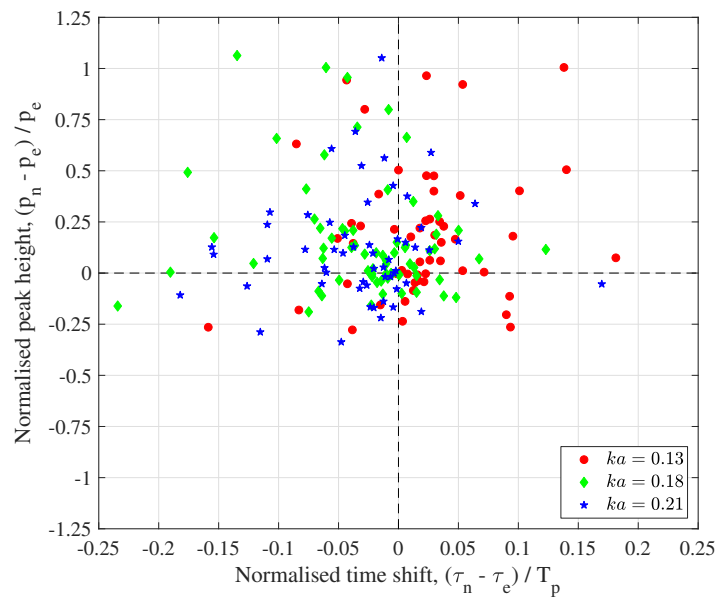

Fig. 16 Normalised peak height vs. normalised time shift for peaks in pressure on the bow, at the SWL, for all participants and cases in Part 1. Colour coded by wave steepness.

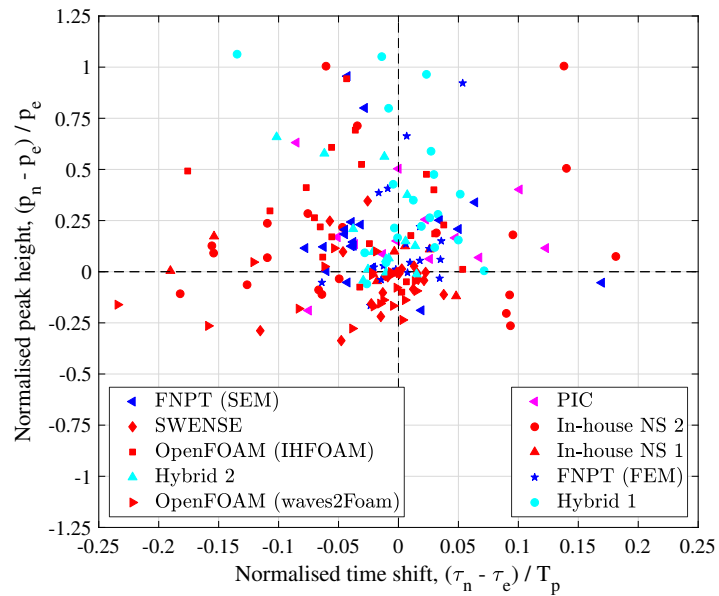

Fig. 17 Normalised peak height vs. normalised time shift for peaks in pressure on the bow, at the SWL, for all participants and cases in Part 1. Colour coded by code type.

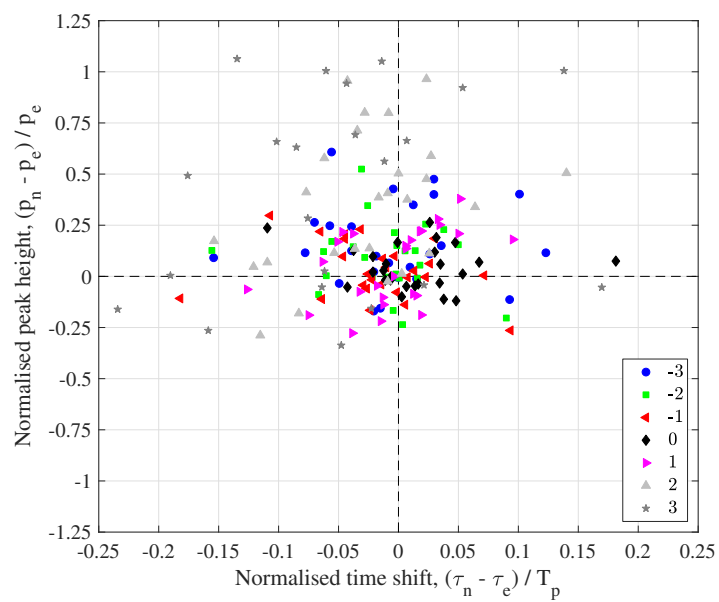

Fig. 18 Normalised peak height vs. normalised time shift for peaks in pressure on the bow, at the SWL, for all participants and cases in Part 1. Colour coded by peak number. 
can be found trivially. However, this kind of reduction inevitably reduces the understanding of the prediction and, without a much greater number of test cases, the confidence in any trends observed, i.e. by ignoring any other inaccuracies in the prediction. How can one be confident that the prediction would be equally good in cases with different conditions (it could just be coincidence that the prediction is good at that particularly moment)? It is perhaps arguable that reducing the comparison to the prediction of a single number, like a maximum, returns the analysis to one that is again case-specific and does little to provide a parametric understanding of the predictive capability a method and thus its routine application by industry. It seems that, once again, in order to generalise the understanding of the predictive capability of numerical models, it is the comparative process that requires a well-thought-out conception.

In this study, Figures 13, 14 and 15 display the normalised peak height (defined as the difference between the numerical prediction and the physical measurement, $\eta_{n}-\eta_{e}$, over the physical result, $\eta_{e}$ ) against the normalised time shift (defined as the difference between the numerical prediction and the physical measurement, $\tau_{n}-\tau_{e}$, over the peak period of the incident wave spectrum, $T_{p}$ ) of peaks in the run-up on the bow (for Part 1), colour-coded by the incident wave steepness, underlying theory of the code used and the peak number respectively (where a peak number of zero corresponds the peak immediately after the main trough and increases with time of occurrence). It can be seen that there is considerable spread in the predicted peak heights and timings and that although the spread in phase discrepancies appears to be even with respect to the physical data, the numerical reproductions typically over estimate the peak heights (as observed in the time series data). From Figure 13, there does not appear to be a relationship between the wave steepness and the quality of the predicted amplitudes but there is a suggestion that in the low steepness case, peaks in run-up lead, while in the high steepness case, the peaks lag the physical data. Figure 14 shows that these trends don't appear to be a function of the code type but many of the low amplitude predictions are from a single code (In-house NS 2) reinforcing the apparent over-estimation in the predicted amplitudes. Figure 14 also suggests that the two hybrid codes are slightly better at predicting the phasing of the peaks. Figure 15 shows that the peaks with the greatest discrepancy in amplitude tend to be those that occur at least two peaks after the main event, i.e. when reflected waves begin to be an issue, and could potentially be disregarded from the assessment.

Figures 16, 17 and 18 show similar scatter plots for the pressure peaks on the bow, at the still water level (for Part 1). The same trends as in the run-up are present, with the additional observation of a wider spread in the phase discrepancies (as noted in qualitative analysis of the time series data in Figures 8 and 9).

These scatter diagrams seem like an effective analysis strategy, they give details of predicted amplitude and phase, for multiple discrete 'events' in each prediction and, with the use of colour-coding, include the key case-specific variables like wave steepness. However to include both the wave steepness and the code type simultaneously is not possible and so further interpretation is required, particularly to form a quantitative comparison. Furthermore, these plots do not offer directly any information regarding the predicted frequency content which may be a critical value in some cases.

\section{CONCLUSIONS}

The CCP-WSI Blind Test Series 1 consists of a series of test cases involving focused wave interactions with a fixed FPSO-like structure. In each case the incident wave remained unbroken and was varied in steepness (Part 1) or angle of incidence (Part 2). The aims of the study are: to assess the numerical codes currently in use (a software audit); provide a better understanding of the required model fidelity in WSI simulations and; to help inform the development of future numerical modelling standards, to encourage the practical application of these tools by industry.

Ten different codes are used in the test, including a range of underlying complexities from FNPT to NS solvers, mesh-based and partially particle methods, high- and low-order methods, and both in-house and open-source codes. Despite considerable scatter in the predictions from 'similar' NS codes (highlighting a real sensitivity and a need for 'best practice' implementation strategies), all cases in the test are generally predicted well by all participating codes. Therefore, in terms of understanding the required model fidelity, the test is inconclusive; the FNPT methods provide good solutions for the (non-violent) cases considered and there is no real benefit in using high-fidelity methods which are considerably more computationally expensive. Furthermore, for the cases considered here, there is no obvious trend in the predictive capability of the codes as a function of either wave steepness or direction. Therefore, in order to find the expected divergence in predictive capability, between FNPT and NS methods, it is suspected that the test cases must cover more violent WSI in which the underlying assumptions of FNPT methods, i.e. inviscid, irrotational flow, are violated. However, it is suspected that including more violent flow phenomena, such as wave breaking, will greatly increase the complexity of the comparisons and the uncertainty in the benchmarking, physical data. Despite this, a significant amount has been learned about the process of performing a comparative study and the requirements on generating the necessary information to establish generalised numerical modelling standards and certification of numerical codes. A discussion on various data analysis/comparison methods and their suitability is given as well as a reflection on the key considerations required when performing such a study.

In conclusion, it is noted that the design of the comparative study is crucial if the results of the study are to provide any conclusive evidence for the predictive capability of the numerical methods. If a parametric understanding is sought, the test cases must be very well conceived and the criteria used for comparing the codes should be understood well and identified in advance. The physical experiments used in the comparison, including any errors/uncertainties, must be understood well. The analysis strategy used must be well conceived and any bias introduced, in the reduction of the results, considered in the comparison. Variations in implementation, such as mesh/domain variations, must also be considered as these can dominate differences in the predicted results.

It is clear that, further work is required to established effective practices for blind tests, and comparative studies, in WSI research and realise the aims of the CCP-WSI Blind Test Workshops. Consequently, a further two CCP-WSI Blind Test Series have been arranged, utilising the recommendations and the findings made here.

\section{ACKNOWLEDGMENTS}

The CCP-WSI Working Group would like to acknowledge the participants of the CCP-WSI Blind Test Series 1 for their contributions as well as the International Society of Offshore and Polar Engineers (ISOPE) and the International Hydrodynamics Committee (IHC) for supporting the Blind Test as part of the ISOPE conference series. The authors would also like to acknowledge the COAST Laboratory staff at the University of Plymouth, UK, for their assistance in producing the physical measurements used in the test. The CCP-WSI is funded by the Engineering and Physical Sciences Research Council (EPSRC) in the UK (EP/M022382/1) and the CCP-WSI Working Group would like to thank them for their continued support of this research. The full physical data set from the CCP-WSI Blind Test Series 1 is available as a long standing test case for future benchmarking and can be downloaded from http://www.ccp-wsi.ac.uk/blind_test_series_1 


\section{REFERENCES}

Brown, S., Musiedlak, P.-H. Ransley, E. \& Greaves, D. (2018). "Numerical simulation of focused wave interactions with a fixed FPSO using OpenFOAM 4.1.", in Proceedings of the 28th International Ocean and Polar Engineering Conference, June 10-15 2018, Sapporo, Japan, pp. 1498-1503, ISBN 978-1-880653-87-6

Buldakov, E. (2013). "Tsunami generation by paddle motion and its interaction with a beach: Lagrangian modelling and experiment.", Coastal Engineering, Vol. 80, pp. 83-94.

Buldakov, E., Stagonas, D., \& Simons, R. (2017). "Extreme wave groups in a wave flume: Controlled generation and breaking onset.", Coastal Engineering, Vol. 128, pp. 75-83.

CCP-WSI (2016). "Wave Structure Interaction Computation and Experiment Roadmap - Part 1: A Report on the 1st CCP-WSI Focus Group Workshop", available online at https://www.ccp-wsi.ac.uk/sites/www.ccp-wsi.ac.uk/files/CCPWSIFocusGroupWS1_Report.pdf

Chen, Q., Zang, J., Kelly, D. M., Dimakopoulos, A. S., (2018). “A 3D parallel particle-in-cell solver for wave interaction with vertical cylinders. ", Ocean Engineering, Vol. 147, pp. 165-180.

Chen, Q. \& Zang, J. (2018). "Numerical modelling of focused wave impact with a fixed FPSO-like structure using a particle-in-cell solver. ", in Proceedings of the 28th International Ocean and Polar Engineering Conference, June 10-15 2018, Sapporo, Japan, pp. 14811485, ISBN 978-1-880653-87-6

Engsig-Karup, A. P., Glimberg, L. S., Nielsen, A. S. and Lindberg, O. (2013). "Fast hydrodynamics on heterogeneous many-core hardware.", Part of: Raphel Couturier. Designing Scientific Applications on GPUs, pp. 251-294, CRC Press / Taylor \& Francis Group, ISBN: 978-1-4665-7162-4

Engsig-Karup, A. P., Eskilsson, C., and Bigoni, D. (2016). “A Stabilised Nodal Spectral Element Method for Fully Nonlinear Water Waves", Journal of Computational Physics, Vol. 318, pp. 1-21.

Engsig-Karup, A.P. and Eskilsson, C. (2018). "Spectral Element FNPF Simulation of Focused Wave Groups Impacting a Fixed FPSO”, in Proceedings of the 28th International Ocean and Polar Engineering Conference, June 10-15 2018, Sapporo, Japan, pp. 1443-1450, ISBN 978-1-880653-87-6

Gatin, I., Jasak, H., Vukĉević V. (2018). "Focused Wave Loading on a Fixed FPSO using Naval Hydro pack", in Proceedings of the 28th International Ocean and Polar Engineering Conference, June 10-15 2018, Sapporo, Japan, pp. 1434-14442, ISBN 978-1-880653-87-6

Hann, M., Greaves, D \& Raby, A. (2015). "Snatch loading of a single taut moored floating wave energy converter due to focussed wave groups", Ocean Engineering, Vol. 96, pp. 258-271.

Higuera, P., Lara, J. L., \& Losada, I. J. (2013). "Realistic wave generation and active wave absorption for NavierStokes models: Application to OpenFOAM ${ }^{\circledR}$ ", Coastal Engineering, Vol. 71, pp. 102-118.

Higuera, P. (2017). “olaFlow”, DOI: 10.5281/zenodo.129701.

Higuera, P., Buldakov, E. \& Stagonas, D. (2018). "Numerical modelling of wave interaction with an FPSO using a combination of OpenFOAM ${ }^{\circledR}$ and Lagrangian models.", in Proceedings of the 28th International Ocean and Polar Engineering Conference, June 10-15 2018, Sapporo, Japan, pp. 1486-1491, ISBN 978-1-880653-87-6

Hong, S. Y., Kim, K.-H., Ha, Y.-J. \& Nam, B. W. (2018). “Comparative Study of Wave Impact Loads on Circular Cylinder by Breaking Waves.", in Proceedings of the 28th International Ocean and Polar Engineering Conference, June 10-15 2018, Sapporo, Japan, pp. 3845, ISBN 978-1-880653-87-6
Jacobsen, N, Fuhrman, D and Fredsøe, J. (2012). "A wave generation toolbox for the open-source CFD library: OpenFOAM ${ }^{\circledR}$, , Int. Journal for Numerical Methods in Fluids, Vol. 70, pp. 1073-1088

Jasak, H., Vukĉević, V., Gatin, I. (2015). "Numerical Simulation of Wave Loads on Static Offshore Structures ", in: CFD for Wind and Tidal Offshore Turbines, Springer Tracts in Mechanical Engineering, pp. 95-105

Li, Q., Zhuang, Y., Wan, D., \& Chen, G. (2018a). "Numerical Analysis of the Interaction between a Fixed FPSO Benchmark Model and Focused Waves", in Proceedings of the 28th International Ocean and Polar Engineering Conference, June 10-15 2018, Sapporo, Japan, pp. 1420-1427, ISBN 978-1-880653-87-6

Li, Q., Yan, S., Wang, J. \& Ma, Q. (2018b). "Numerical Simulation of Focusing Wave Interaction with FPSO-like Structure Using FNPTNS Solver", in Proceedings of the 28th International Ocean and Polar Engineering Conference, June 10-15 2018, Sapporo, Japan, pp. 1458-1464, ISBN 978-1-880653-87-6

Ma, Q. W., \& Yan, S. (2006). "Quasi ALE finite element method for nonlinear water waves ", Journal of computational physics, Vol.212(1), pp. 52-72.

Mai, T., Greaves, D., Raby, A. \& Taylor, P. (2016). "Physical modelling of wave scattering around fixed FPSO-shaped bodies", Applied Ocean Research, Vol. 61, pp. 115-129.

Musiedlak, P-H., Ransley, E., Greaves, D., Child, B., Hann, M. \& Iglesias, G. (2017). "Investigations of model validity for numerical survivability testing of WECs", Proceedings of the $12^{\text {th }}$ European Wave and Tidal Energy Conference (EWTEC), Cork, Ireland.

National Maritime Research Institute, Tokyo, Japan (NMRI) (2015). "Tokyo 2015: a Workshop on CFD in Ship Hydrodynamics ", available online at http://www.t2015.nmri.go.jp/index.html

Shen, Z. R., Zhao, W. W., Wang, J. H. \& Wan, D. C. (2014). “Manual of CFD solver for ship and ocean engineering flows: naoe-FOAMSJTU ", Technol Rep Solver Man, Shanghai Jiao Tong University.

Tromans, P. S., Anaturk, A. R., \& Hagemeijer, P. (1991). “A new model for the kinematics of large ocean waves - application as a design wave ", in Proceedings of the 1st International Offshore and Polar Engineering Conference, Edinburgh, UK, pp. 64-71

Vukĉević, V., Jasak, H., Malenica, S. (2016). "Decomposition model for naval hydrodynamic applications, Part I: Computational method ", Ocean Engineering, Vol. 121, pp.37-46

Vukĉević, V., Jasak, H., Gatin, I. (2017). "Implementation of the Ghost Fluid Method for free surface flows in polyhedral Finite Volume framework ", Computers $\mathcal{F}$ Fluids, Vol. 153, pp. 1-19

Xie, Z. (2012). "Numerical study of breaking waves by a two-phase flow model ", International Journal for Numerical Methods in Fluids, Vol. 70(2), pp. 246-268

Xie, Z. (2015). "A two-phase flow model for three-dimensional breaking waves over complex topography ", Proceedings of the Royal Society A: Mathematical, Physical $\mathcal{F}$ Engineering Sciences, Vol. 471: 20150101

Xie, Z., Yan, S., Ma, Q., \& Stoesser, T. (2018). "Numerical modelling of focusing wave impact on a fixed offshore structure. ", in Proceedings of the 28th International Ocean and Polar Engineering Conference, June 10-15 2018, Sapporo, Japan, pp. 1451-1457, ISBN 978$1-880653-87-6$

Yan, S., \& Ma, Q. W. (2010). "QALEFEM for modelling 3D overturning waves ", International Journal for Numerical Methods in Fluids, Vol. 63(6), pp. 743-768. 